\title{
Gluino mass limits with sbottom NLSP in coannihilation scenarios
}

\author{
Arindam Chatterjee, ${ }^{a}$ Arghya Choudhury, ${ }^{a}$ Amitava Datta ${ }^{b}$ and \\ Biswarup Mukhopadhyaya ${ }^{a}$ \\ ${ }^{a}$ Regional Centre for Accelerator-based Particle Physics, Harish-Chandra Research Institute, \\ Allahabad 211019, India \\ ${ }^{b}$ Department of Physics, University of Calcutta, \\ 92 A.P.C. Road, Kolkata 700 009, India \\ E-mail: arindam@hri.res.in, arghyachoudhury@hri.res.in, \\ adatta@iiserkol.ac.in, biswarup@hri.res.in
}

ABSTRACT: In view of the recent interest in the pMSSM with light third generation squarks, we consider a hitherto neglected scenario where the lighter bottom squark $\left(\widetilde{b}_{1}\right)$ is the next lightest supersymmetric particle (NLSP) which co-annihilates with the lightest supersymmetric particle (LSP), the dark matter (DM) candidate. Since the co-annihilation cross section receives contributions from both electroweak and strong vertices, it is relatively large. As a result relatively large NLSP-LSP mass difference $(25-35 \mathrm{GeV})$ is consistent with the PLANCK data. This facilitates the LHC signatures of this scenario. We consider several variants of the sbottom NLSP scenario with and without light stops and delineate the parameter space allowed by the PLANCK data. We point out several novel signal (e.g., $\left.\widetilde{t}_{1} \rightarrow \widetilde{b}_{1} W\right)$ which are not viable in the stop NLSP scenario of DM production. Finally, we consider gluino $(\widetilde{g})$ decays in this scenario and using the current ATLAS data in the jets (with or without b-tagging) $+\mathbb{H}_{T}$ channel, obtain new limits in the $m_{\widetilde{b}_{1}}-m_{\widetilde{g}}$ mass plane. We find that for $m_{\widetilde{b}_{1}}$ upto $500 \mathrm{GeV}, m_{\widetilde{g}} \geq 1.1-1.2 \mathrm{TeV}$ in this scenario.

KEYWORDS: Supersymmetry Phenomenology

ARXiv EPRINT: 1411.6467 


\section{Contents}

1 Introduction 1

2 Co-annihilating $\tilde{b}_{1}$ and $\widetilde{\chi}_{1}^{0}$ relic abundance: with and without light stop 3

3 Constraints on the $\widetilde{b}_{1}-\widetilde{g}$ sector in the $\widetilde{b}_{1}$ NLSP scenarios 9

4 Conclusion $\quad 13$

\section{Introduction}

Supersymmetry (SUSY) [1-5] is the most popular and widely studied extension of the standard model (SM) of particle physics. In the R-parity conserving minimal supersymmetric standard model (MSSM), the lightest neutralino is the lightest SUSY particle (LSP) and a viable dark matter (DM) [6-11] candidate. R-parity makes the LSP stable leading to missing energy $\left(\mathscr{F}_{T}\right)$ signals at the Large Hadron Collider (LHC). Right from the beginning of the LHC run both the ATLAS and CMS collaborations are looking for SUSY using this feature of the signals in various channels. In the absence of any excess events they have put stringent bounds on the masses of supersymmetric particles (sparticles) from the 7 and $8 \mathrm{TeV}$ data. The limits on the masses of strongly interacting sparticles are the strongest, due to the to large production cross-sections [12-19]. For example, at the end of the $8 \mathrm{TeV}$ run, $m_{\text {squark }}\left(m_{\tilde{q}}\right)=m_{\text {gluino }}\left(m_{\tilde{g}}\right)$ scenarios are ruled out upto $1.7 \mathrm{TeV}$ [12].

It should be borne in mind that comprehensive SUSY search strategies in the upcoming experiments at LHC $13 \mathrm{TeV}$ runs will be designed on the basis of the exclusions obtained by the experiments during the first phase. It is, therefore, worthwhile to revisit the limits critically and find out the models in which some loopholes in the current search techniques significantly relax the limits. A case in point is the compressed SUSY scenario which can considerably relax the limits [20-26].

In view of the stringent bounds on the first two generation squark masses, models with light third generation have received much attention in recent times [27-51]. It may be recalled that such heavy squarks offer a way of ameliorating the SUSY flavour [52] and CP problems $[53,54]$. Such scenarios also help in restoring the naturalness of a SUSY model.

In this analysis, we focus on scenarios with a light $\widetilde{b}_{1}$, both with and without a $\widetilde{t}_{1}$, along with a heavier gluino which exclusively decays into these squarks. We further assume that the remaining members of the MSSM spectrum are heavy, except the lightest neutralino which lies below the $\widetilde{b}_{1}$. The $\widetilde{b}_{1}$ is the next-to-lightest SUSY particle (NLSP) in this scenario.

An added attraction, and at the same time a crucial viability check of this spectrum is that LSP-NLSP coannihilation can produce the observed DM relic density of the universe 
provided their mass difference is small. A large number of phenomenological analyses have already addressed various topics/issues related to stop searches in different channels [27$42]$ and investigated DM production via $\widetilde{t}_{1}-\widetilde{\chi}_{1}^{0}$ coannihilation [37-42] extensively in the context of LHC. On the other hand the sbottom NLSP scenario, which also has the potential of explaining the observed relic density, has not yet received due attention and has been addressed by a relatively small number of analyses only [43-51]. Moreover, most of the analyses predating the first phase of the LHC experiments were restricted to mSUGRA motivated models with non-universal boundary conditions. Since the SUSY breaking mechanism is essentially unknown, our emphasis will be on the $\widetilde{b}_{1}-\widetilde{\chi}_{1}^{0}$ coannihilation scenario in the phenomenological minimal supersymmetric standard model (pMSSM) [55] constrained by the LHC data. Thus, clear perspectives are expected to emerge from this analysis on (a) the viability of sbottom-neutralino coannihilation (with or without the assistance of stops) from the standpoint of relic density, (b) the spectacular signatures, not viable in the $\widetilde{t}_{1}$-NLSP scenario, expected in the phase 2 of the LHC experiments and c) the new constraints in the $\widetilde{b}_{1}-\widetilde{g}$ sector in the $\widetilde{b}_{1}-\widetilde{\chi}_{1}^{0}$ co-annihilation scenarios using the current LHC data.

It should be noted in addition that one of the scenarios investigated here, namely the one with just a (right) $\widetilde{b}_{1}$ and both stop mass eigenstates heavy, has an appeal from the viewpoint of the lighter neutral Higgs mass. It is known that pushing this mass upto $\approx$ $125 \mathrm{GeV}$ becomes less troublesome if the stop mass(es) and the trilinear SUSY breaking term is large. It therefore helps to have a situation where both of the stop eigenstates participate in raising the Higgs mass, while a light $\widetilde{b}_{1}$ lies close to the lightest neutralino and and facilitates co-annihilation.

Before delving into our main analysis it is worthwhile to review the existing LHC limits on $m_{\widetilde{t}_{1}}$ and $m_{\widetilde{b}_{1}}$ from direct search of these sparticles. If $\Delta m_{\widetilde{t}_{1}}=m_{\widetilde{t}_{1}}-m_{\widetilde{\chi}_{1}^{0}}$ is relatively large so that the decay $\widetilde{t}_{1} \rightarrow t \widetilde{\chi}_{1}^{0}$ decay is allowed, stop masses in the range 210-650 GeV are excluded for $m_{\widetilde{\chi}_{1}^{0}} \lesssim 30 \mathrm{GeV}[56,57]$. As $m_{\widetilde{\chi}_{1}^{0}}$ increases the limits become weaker. Similarly from sbottom pair production $m_{\widetilde{b}_{1}}<620 \mathrm{GeV}$ is excluded at $95 \%$ CL for $m_{\widetilde{\chi}_{1}^{0}} \lesssim 150 \mathrm{GeV}$ [58]. However, for $m_{\widetilde{\chi}_{1}^{0}} \gtrsim 250 \mathrm{GeV}$ there is no limit on $m_{\widetilde{t}_{1}}$ and $m_{\widetilde{b}_{1}}$.

None of the above scenarios, however, is consistent with the observed DM relic density of the universe. To explain the correct relic density, we need small mass difference between LSP and the NLSP (stop/sbottom) and a different technique for the NLSP search is called for. The limits on masses on third generation squarks are remarkably weaker in such coannihilating scenarios. From direct stop pair production the latest bound on $m_{\widetilde{t}_{1}}$ is $240 \mathrm{GeV}$ [59] for $m_{\widetilde{t}_{1}}-m_{\widetilde{\chi}_{1}^{0}}<80 \mathrm{GeV}$. If the stop and the lightest neutralino are almost degenerate then stop masses up to $260 \mathrm{GeV}$ are excluded from ATLAS search with a 'monojet like' topology ${ }^{1}$ [59]. Very recently using this search channel and $20 \mathrm{fb}^{-1}$ data at $8 \mathrm{TeV}, m_{\widetilde{b}_{1}}$ below $250 \mathrm{GeV}$ is excluded for small $\Delta m_{\widetilde{b}_{1}}=m_{\widetilde{b}_{1}}-m_{\widetilde{\chi}_{1}^{0}}[59]$.

As the gluino pair production cross-sections is the largest, gluino decays into the third generation squarks are likely to probe larger ranges of $m_{\widetilde{t}_{1}}$ and/or $m_{\widetilde{b}_{1}}$ in the coannihilation

\footnotetext{
${ }^{1}$ Here one depends on a hard ISR jet in enhancing the signal. However, the signal with low jet multiplicity may contain more than one jet.
} 
scenario. Many groups have looked for gluino decay signatures in presence of light third generation squarks [60-68]. In such cases gluino decays into $\widetilde{t}_{1} t$ and/or $\widetilde{b}_{1} b$. For coannihilating scenarios $\widetilde{t}_{1}$ decays into $c \widetilde{\chi}_{1}^{0}$ and $\widetilde{b}_{1}$ decays into $b \widetilde{\chi}_{1}^{0}$ with $100 \%$ BRs. However the $c$ or $b$ jets coming from $\widetilde{t}_{1}$ or $\widetilde{b}_{1}$ will be softer and the limits on the gluino masses could be degraded. For example, if all three generations squarks are much heavier than the gluino, then $\operatorname{BR}\left(\widetilde{g} \rightarrow q \bar{q} \widetilde{\chi}_{1}^{0}\right)$ is $100 \%$ where $q$ is any quark. In this case gluino masses below $1.4 \mathrm{TeV}$ is excluded for $m_{\widetilde{\chi}_{1}^{0}} \lesssim 200 \mathrm{GeV}$ from jets $+\mathbb{F}_{T}$ channel [12]. On the other hand assuming $m_{\widetilde{t}_{1}}-m_{\widetilde{\chi}_{1}^{0}}=20 \mathrm{GeV}$ which is relevant for the $\widetilde{t}_{1}$ - LSP coannihilation and $\operatorname{Br}\left(\widetilde{g} \rightarrow \widetilde{t}_{1} t \rightarrow c t \widetilde{\chi}_{1}^{0}\right)=100 \%$, the limit reduces to $1150 \mathrm{GeV}[12]$. The ATLAS collaboration have also updated their search for NLSP sbottom scenario in the $0-1 l+3 b-j$ ets $+\mathbb{H}_{T}$ channel [13] with $\operatorname{Br}\left(\widetilde{g} \rightarrow \widetilde{b}_{1} b \rightarrow b b \widetilde{\chi}_{1}^{0}\right)=100 \%$ and have excluded $m_{\widetilde{g}}$ below $1.2 \mathrm{TeV}$ upto $m_{\widetilde{b}_{1}}=1.0 \mathrm{TeV}$. But the model is in conflict with the DM relic density as the LSP mass is fixed at $60 \mathrm{GeV}$.

In this paper we have revisited the last analysis focusing on $\Delta m_{\widetilde{b}_{1}}$ appropriate for the coannihilation scenario, and checked whether this relaxes the gluino mass limit. We have considered several variants of the sbottom NLSP scenario characterized by the presence or absence of a $\widetilde{t}_{1}$. In contrast, the experimental searches have considered gluino decays either into the $\widetilde{t}_{1}$ or the $\widetilde{b}_{1}$ channel. But if both $\widetilde{t}_{1}$ and $\widetilde{b}_{1}$ are light, then, depending on their mass difference, the gluino may decay into both the channels with sizable BRs. If, for example, the stop exclusively decays into a soft charm jet the gluino mass limit may suffer further suppression. Moreover the presence of an additional light $\widetilde{t}_{1}$ predicts novel signal for the LHC phase 2 experiments as we shall elaborate below.

An optimized search strategy for the $\widetilde{b}_{1}$ coannihilation scenario in the LHC phase 2 experiments calls for immediate attention. In this paper we use the existing ATLAS search results to obtain the best limits available at the moment in this scenario with/without a light stop. We also compare these results with the ones obtained by a generic jets $+\mathbb{F}_{T}$ search for strongly interacting sparticles.

In section 2 , we discuss the variants of the scenario studied here, and identify the regions of the parameter space which are consistent with the observed relic density as well as direct DM search experiments. We also identify the spectacular signatures in the $\widetilde{b}_{1}$ NLSP scenario in presence of a relatively light $\widetilde{t}_{1}$. The new constraints in the $m_{\widetilde{b}_{1}}-m_{\widetilde{g}}$ plane in different scenarios using the LHC Run I data are computed in section 3. We summarize our main conclusions in section 4 .

\section{Co-annihilating $\tilde{b}_{1}$ and $\widetilde{\chi}_{1}^{0}$ relic abundance: with and without light stop}

We explore a (simplified) pMSSM scenario with a bino-like LSP $\left(\widetilde{\chi}_{1}^{0}\right)$ and $\widetilde{b}_{1}$ as NLSP. Since the bino couples to the other (s)particles only through $\mathrm{U}(1)_{y}$ gauge interaction, the annihilation cross-section into standard model particles is known to be small. As a consequence, a (pure) bino-like $\widetilde{\chi}_{1}^{0}$ decouples from the thermal soup sufficiently early, and therefore has a larger thermal relic abundance compared to the present measured relic abundance $\Omega h^{2}=0.112$ [69] over large region of the parameter space. 
However, when there is a scope for co-annihilation with the NLSP the DM and the coannihilating sparticle $\left(\widetilde{b}_{1}\right.$ in this case) remain in relative thermal equilibrium for a longer period of time through $\widetilde{\chi}_{1}^{0} S M \leftrightarrow \widetilde{b}_{1} S M^{\prime}$, where $S M$ and $S M^{\prime}$ denote two Standard Model particles which are assumed to be in thermal equilibrium and therefore abundant. In the context of a bino-like $\widetilde{\chi}_{1}^{0}$, the $s$-channel $\widetilde{b}_{1}$ exchange process $\widetilde{\chi}_{1}^{0} b \leftrightarrow \widetilde{b}_{1} g$, and the $t / u$ channel $b$ exchange processes $\widetilde{\chi}_{1}^{0} g \leftrightarrow b \widetilde{b}_{1}$ are examples of this. Thus, larger (co-)annihilation cross-section of $\widetilde{b}_{1}$ also implies a lower thermal relic density of the $\widetilde{\chi}_{1}^{0}$. Of course, these interactions eventually decouple and the remaining sbottoms decay to $\widetilde{\chi}_{1}^{0}$. The effect can be captured by [70, 71] using an effective (co-)annihilation cross-section $\left(\sigma_{\text {eff }}\right)$,

$$
\sigma_{\text {eff }}=\Sigma_{i, j} \frac{g_{i} g_{j}}{g_{\text {eff }}^{2}}\left(1+\Delta_{i}\right)^{3 / 2}\left(1+\Delta_{j}\right)^{3 / 2} e^{-x\left(\Delta_{i}+\Delta_{j}\right)} \sigma_{i j},
$$

instead of the annihilation cross-section $\sigma_{a n n}$ in the relevant expressions. In the above equation, $\{i, j\}$ runs over the list of co-annihilating sparticles, $g_{i}$ denotes the number of degrees of freedom of the $i$-th sparticle, $\Delta_{i}=\frac{m_{i}}{m_{\widetilde{\chi}_{1}^{0}}}-1, x=\frac{m_{\widetilde{\chi}_{1}^{0}}}{T}$ and $\sigma_{i j}$ denotes the co-annihilation cross-section of $i$ and $j$-th sparticles into SM particles. Also,

$$
g_{\mathrm{eff}}=\Sigma_{i} g_{i}\left(1+\Delta_{i}\right)^{3 / 2} e^{-x \Delta_{i}} .
$$

Thus co-annihilations are very relevant for sufficiently small $\Delta_{i}$, i.e. for small mass difference between the LSP and the NLSP. In this paper we choose the mass difference between $\widetilde{\chi}_{1}^{0}$ and $\tilde{b}_{1}$ such that sufficient co-annihilation takes place [70, 71] ensuring the correct thermal relic abundance of $\widetilde{\chi}_{1}^{0}$. Note that, LSP co-annihilation with strongly interacting sparticles the involves one strong vertex leading to large cross-section. Consequently to achieve the required $\sigma_{\text {eff }}$, the mass difference of these strongly interacting sparticles with the LSP need to be larger compared to their electroweak counterparts in a co-annihilation scenario. As we will see, while the required mass difference is about $5-15 \mathrm{GeV}$ for electroweak sparticles (see, e.g., $[72,73]$ ), for (third generation) squarks it is about $25-35 \mathrm{GeV}$. This has nontrivial consequences for the observability of $\widetilde{b}_{1}$ coannihilation scenario at the LHC (to be discussed in the next section).

In the pMSSM, the soft-supersymmetry-breaking mass terms for the third generation squarks, namely $m_{\tilde{b}_{R}}\left(m_{\tilde{t}_{R}}\right)$ for the $\mathrm{SU}(2)_{L}$ singlets $\tilde{b}_{R}\left(\tilde{t}_{R}\right)$ and $m_{\tilde{Q}_{3}}$ for the $\mathrm{SU}(2)_{L}$ doublets are free parameters. A large hierarchy among the parameters $m_{\tilde{b}_{R}}\left(\tilde{t}_{R}\right)$ and $m_{\tilde{Q}_{3}}$ ensure that L-R mixing in the sbottom ( stop ) sector is small. We shall restrict ourselves to such scenarios only. We further assume that the first two generations of squarks are much heavier and decoupled. The electroweak sparticles other than the LSP are assumed to be heavier than $\widetilde{b}_{1}$ or $\widetilde{t}_{1}$. We consider three $\widetilde{b}_{1}$-LSP coannihilation scenarios characterized by different relative magnitudes of the above soft terms leading to potentially distinctive LHC signatures. Each scenario is illustrated by a BM point in table 1 and the corresponding BRs relevant for LHC signatures are tabulated in table 2 .

- Gluino-Sbottom-R-Model: Here the $\tilde{b}_{1} \mathrm{NLSP}$ is a $\mathrm{SU}(2)_{L}$ singlet with a small $m_{\tilde{b}_{R}}$. The parameters $m_{\tilde{Q}_{3}}$ and $m_{\tilde{t}_{R}}$ are assumed to be too large to affect the gluino decays. Benchmark point (BMP) BMP-A in table 1 provides an example of this scenario. 


\begin{tabular}{|c|c|c|c|c|c|}
\hline Parameter & BMP-A & BMP-B1 & BMP-B2 & BMP-B3 & BMP-C \\
\hline$m_{\widetilde{\chi}_{1}^{0}}$ & 300 & 306 & 305 & 305 & 305 \\
$m_{\widetilde{g}}$ & 1236 & 1259 & 1273 & 1270 & 1310 \\
$m_{\widetilde{b}_{1}}$ & 325 & 335 & 333 & 333 & 334 \\
$m_{\widetilde{t}_{1}}$ & 1558 & 345 & 507 & 468 & 455 \\
\hline$\Omega_{h}^{2}$ & 0.11 & 0.11 & 0.11 & 0.11 & 0.12 \\
\hline
\end{tabular}

Table 1: Mass spectra for different benchmark points. BMP-A,B(1,2,3),C represent three different scenarios Gluino-Sbottom-R-Model, Gluino-Stop-Sbottom-R-Model and GluinoStop-Sbottom-L-Model respectively (see text for details). All the masses are in GeV.

- Gluino-Stop-Sbottom-R-Model: In this model the NLSP is dominantly $\tilde{b}_{R}$ as in the previous case. However, there is also a R-type light $\widetilde{t}_{1}$. In contrast to the previous scenario, $\tilde{g}$ decays to both $b \widetilde{b}_{1}$ and $t \widetilde{t}_{1}$ channels with sizable BRs. Depending on the mass splitting between $\widetilde{b}_{1}$ and $\widetilde{t}_{1}$, there are three possible decay modes of $\widetilde{t}_{1}: c \widetilde{\chi}_{1}^{0}$, $t \widetilde{\chi}_{1}^{0}$ and $\widetilde{b}_{1} W$. They are tabulated as BMP-B1, BMP-B2 and BMP-B3 respectively in table 1.

If the mass difference between $\widetilde{b}_{1}$ and $\widetilde{t}_{1}$ is assumed to be significantly smaller than $m_{W}, \widetilde{t}_{1}$ decays dominantly into the loop induced mode $c \widetilde{\chi}_{1}^{0}$. As a result the number of taggable hard jets in the signal decreases and this scenario is expected to yield the weakest limits on $m_{\widetilde{g}}$. Moreover, both $\widetilde{t}_{1}$ and $\widetilde{b}_{1}$ can contribute to DM production if their mass difference is $10 \mathrm{GeV}$ or so. BMP-B1 in the table illustrates this case. However, if $m_{\widetilde{t}_{1}} \gtrsim m_{t}+m_{\widetilde{\chi}_{1}^{0}}$, then $\widetilde{t}_{1} \rightarrow t \widetilde{\chi}_{1}^{0}$ dominates; BMP-B2 illustrates this scenario. Finally, for $m_{W}+m_{\widetilde{b}_{1}} \lesssim m_{\widetilde{t}_{1}} \lesssim m_{t}+m_{\widetilde{\chi}_{1}^{0}}, \widetilde{t}_{1} \rightarrow \widetilde{b}_{1} W$ dominates over the flavor-violating decay mode $c \widetilde{\chi}_{1}^{0}$ provided there is a tiny L-R mixing in the stop and sbottom sectors. This is depicted by BMP-B3. Note that in this case the L-components in $\widetilde{t}_{1}$ and $\widetilde{b}_{1}$ are respectively enhanced to about $5 \%$ and $0.2 \%$ only by adjusting the parameters in the stop and sbottom mass matrices. However, if both the mode $\widetilde{t}_{1} \rightarrow t \widetilde{\chi}_{1}^{0}$ and $\widetilde{t}_{1} \rightarrow \widetilde{b}_{1} W$ are kinematically allowed the mixing angle suppressed latter mode is not competitive. In the presence of large mixing in the sbottom and/or stop sector the above classifications become somewhat blurred. In this case, e.g, both $\widetilde{t}_{1} \rightarrow \widetilde{b}_{1} W$ and $\widetilde{t}_{1} \rightarrow t \widetilde{\chi}_{1}^{0}$ may be observed, if kinematically allowed. In fact the relative rates of these two events may provide some information on L-R mixing in the squark sector.

- Gluino-Stop-Sbottom-L-Model: In this case only $m_{\tilde{Q}_{3}}$ is relatively light. The $\widetilde{b}_{1}$ NLSP is dominantly an $\mathrm{SU}(2)_{L}$ doublet i.e, of L-type. In the limit of small $L-R$ mixing, $\tilde{t}_{1}$ is heavier than $\tilde{b}_{1}$ due to larger contributions from the D-terms and F-terms. The mass difference between $\widetilde{t}_{1}$ and $\widetilde{b}_{1}$ is fixed and only allows the decay $\tilde{t}_{1} \rightarrow \tilde{b}_{1} W^{+}$ which occurs with $100 \%$. The BMP-C in table 1 illustrates this case.

The BRs relevant for LHC signatures are tabulated in table 2. In all scenarios, $\operatorname{Br}\left(\widetilde{b}_{1} \rightarrow\right.$ 


\begin{tabular}{|c|c|c|c|c|c|}
\hline Decay Modes & BMP-A & BMP-B1 & BMP-B2 & BMP-B3 & BMP-C \\
\hline$\widetilde{g} \rightarrow b \widetilde{b}_{1}$ & 100 & 52.3 & 52.2 & 50.8 & 54.4 \\
$\rightarrow t \widetilde{t}_{1}$ & - & 47.7 & 47.8 & 49.2 & 45.6 \\
\hline$\widetilde{b}_{1} \rightarrow b \widetilde{\chi}_{1}^{0}$ & 100 & 100 & 100 & 100 & 100 \\
\hline$\widetilde{t}_{1} \rightarrow c \widetilde{\chi}_{1}^{0}$ & - & 97 & - & - & - \\
$\rightarrow W \widetilde{b}_{1}$ & - & $3^{*}$ & 1.4 & 100 & 100 \\
$\rightarrow t \widetilde{\chi}_{1}^{0}$ & - & - & 98.6 & - & - \\
\hline
\end{tabular}

Table 2: Branching Ratios - BRs (\%) of decay modes of $\widetilde{g}, \widetilde{b}_{1}$ and $\widetilde{t}_{1}$ for benchmark points. * For BMP-B1 small BRs of $\widetilde{t}_{1} \rightarrow W \widetilde{b}_{1}$ is coming from virtual $W$.

$\left.b \widetilde{\chi}_{1}^{0}\right)$ is $100 \%$, as $\widetilde{b}_{1}$ is the NLSP. In the scenarios with a light $\widetilde{t}_{1}$ the $\tilde{g}$ decays to both $b \widetilde{b}_{1}$ and $t \widetilde{t_{1}}$. For the scenarios represented by BMP-B1, BMP-B3 and BMP-C the BRs of the two modes are approximately the same irrespective of $m_{\widetilde{t}_{1}}$. In BMP-B2 the BRs of the final states with $\widetilde{t}_{1}$ decreases as $m_{\widetilde{t}_{1}}$ approaches $m_{\widetilde{g}}$ and the scenario is indistinguishable to Gluino-sbottom-R model. We have chosen BMP-B2 such that $m_{\widetilde{t}_{1}}$ the $\widetilde{g}$ decays to both modes with approximately the same BR. As discussed above, in the limit of small L-R mixing $\widetilde{t}_{1}$ decays into a single channel channel with almost $100 \% \mathrm{BR}$ for $m_{\widetilde{t}_{1}}$ in a specific range. BMP-B1 and BMP-B2 represent scenarios with qualitatively different stop decays and can in principle be distinguished from other scenarios. On the other hand BMP-B3 and BMP-C are indistinguishable so far as stop decays are concerned.

As already noted no dedicated search for the $\widetilde{b}_{1}$ NLSP has so far been carried out at the LHC. In section 3 we shall focus on new constraints on $m_{\widetilde{b}_{1}}$ and $m_{\widetilde{g}}$ using the available data from phase 1 of the LHC run. However it should be emphasized that spectacular signatures of gluino decays in the $\widetilde{b}_{1}$ NLSP scenario with a light $\widetilde{t}_{1}$ will be worth searching at the LHC during phase 2. Final state topologies like $2 b W \mathbb{F}_{T}, b t W \mathbb{F}_{T}$ or $b t \mathbb{F}_{T}$ are not viable in the $\widetilde{t}_{1}$ - NLSP scenario.

We ensure that the spectrum, generated by SuSpect [74], contains a CP-even (light) Higgs with mass $125 \pm 3 \mathrm{GeV}$, as required by the present LHC data [75-78]. The relevant modes for $\widetilde{g}, \widetilde{b}_{1}$ and $\widetilde{t}_{1}$ decays with their branching ratios (BRs) for the benchmark points are shown in table 2. We have used SUSYHIT [79] to obtain the decay widths and branching ratios in various channels. We have computed the DM relic density using micrOMEGAs-3.6.7. [80].

For the Gluino-Sbottom-R-Model, in estimating the thermal abundance, three processes play major roles. The first one is $\widetilde{b} \widetilde{b}_{1}^{*} \rightarrow g g$, which contributes dominantly to $\sigma_{\text {eff }}$. This process receives contributions from four-point (gauge) interaction, $s$-channel $g$ exchange, and $t / u$-channel $\tilde{b}$ exchange processes where the first two channels contribute most. In addition, there can be annihilation via $\widetilde{\chi}_{1}^{0} \widetilde{b}_{1} \rightarrow g b$ and $\widetilde{b}_{1} \widetilde{b}_{1} \rightarrow b b$. Note that $\widetilde{\chi}_{1}^{0}$ annihilation is a pure EW process and hence its contribution remains small. While $s$-channel $b$ exchange and $t / u$-channel $\widetilde{b}_{1}$ exchange contributes to the former one; the latter one is mediated by $\widetilde{g}$ and neutralinos (mostly the bino-like one) in $t / u$-channel. For our BMP-A, these three 
processes contribute $63 \%, 18 \%$ and $11 \%$ respectively. Although, because of large $m_{\widetilde{g}}$ mass, the contribution from the third process is rather small for smaller $\widetilde{\chi}_{1}^{0}$ masses, it increases for larger $\widetilde{\chi}_{1}^{0}$ masses contributing about $20 \%$ for $m_{\widetilde{\chi}_{1}^{0}} \sim 600 \mathrm{GeV}$.

In the Gluino-Stop-Sbottom-R-Model, for BMP-B1, $\widetilde{b}_{1} \widetilde{b}_{1}^{*} \rightarrow g g$ contributes about $33 \%$; $\widetilde{t}_{1} \widetilde{b}_{1} \rightarrow g b$ and $\widetilde{\chi}_{1}^{0} \tilde{t}_{1} \rightarrow g t$ contribute about $15 \%$ each; and $\widetilde{\chi}_{1}^{0} \widetilde{\chi}_{1}^{0} \rightarrow t \bar{t}$ contributes about $11 \%$. The latter receives contributions from $\tilde{t}_{1}$ exchange $t / u$-channel processes, and is unsuppressed due to the large top mass which helps in evading large chirality suppression. Any other channel contributes less than $10 \%$ for this chosen benchmark point. In BMP-B2 and BMP-B3, $\widetilde{t}_{1}$ is quite heavy compared to $\widetilde{\chi}_{1}^{0}$. Consequently, the leading co-annihilation channels involve $\widetilde{b}_{1}$ only, and their relative contributions are similar to that in GluinoSbottom-R-Model (BMP-A). However, since $\widetilde{t}_{1}$ is quite light compared to that in BMP-A, $\widetilde{\chi}_{1}^{0}$ annihilation into $t \bar{t}$ (mediated via $\widetilde{t}_{1}$ in $t / u$-channel) contributes about $5-6 \%$ in both these cases.

Finally, in the Gluino-Stop-Sbottom-L-Model, the leading contribution comes from $\widetilde{b}_{1} \widetilde{b}_{1}^{*} \rightarrow g g$, which gives about $34 \%$ of the total coannihilation rate for BMP-C. Since $\widetilde{b}_{1}$ is L-type, large D-term contribution together with contributions from the F-term, leads to large $\widetilde{b}_{1} \widetilde{b}_{1}^{*} \rightarrow h h$ cross-section. Along with four-point vertices, $s$-channel $h$ mediated process and $t / u$-channel $\widetilde{b}_{1}$ mediated processes contribute to this channel. Altogether, its contribution is about $22 \%$. This is followed by the cross-sections for $\widetilde{b}_{1} \widetilde{b}_{1} \rightarrow b b$, and other channels which contribute less than $10 \%$ for BMP-C. Since $m_{\tilde{t}_{1}}$ is quite large (due to large D-term and F-term contribution, in the no-mixing limit), co-annihilation with $\tilde{t}_{1}$ does not take place.

In figure 1 , the red points show the region where $\widetilde{\chi}_{1}^{0}$ has the right thermal relic abundance in Gluino-Sbottom-R-Model. The mass difference with $\tilde{b}_{1}$ does not change significantly over a large region of $m_{\widetilde{\chi}_{1}^{0}}$; it varies between about $25-35 \mathrm{GeV}$. Since strong interaction processes involving $\widetilde{b}_{1}$ always contribute dominantly to the relic density, such a minor variation of $\left(m_{\widetilde{b}_{1}}-m_{\widetilde{\chi}_{1}^{0}}\right)$ is well anticipated, especially since $\sigma_{\text {eff }}$ depends exponentially on the same. The figure further shows the present ATLAS limit on $m_{\tilde{b}_{1}}$ derived from $0 l+2 b+\mathbb{E}_{T}$ and $0 l+1 j+\mathbb{E}_{T}$ (compressed scenario) for direct $\widetilde{b}_{1}$ production [58, 59].

In Gluino-Stop-Sbottom-R-Model $\tilde{t}_{1}$ (R-type) also contributes to the co-annihilation processes; for BMP-B1 its contribution is about $30 \%$. Correspondingly, the mass difference $\left(m_{\widetilde{b}_{1}}-m_{\widetilde{\chi}_{1}^{0}}\right)$ is raised by about $4 \mathrm{GeV}$ compared to that in Gluino-Sbottom-R-Model scenario. The mass difference varies between about $28-35 \mathrm{GeV}$ over a large range (up to about $800 \mathrm{GeV}$ ) of $m_{\widetilde{\chi}_{1}^{0}}$ in this scenario too.

Green (blue) Points in figure $2 \mathrm{a}(2 \mathrm{~b})$ represent the parameter space with correct thermal relic density allowed by PLANCK in the Gluino-Stop-Sbottom-R(L)-Model. Orange and pink shaded regions are excluded from direct $\widetilde{b}_{1}$ pair production data in $0 l+2 \mathrm{~b}+\not \mathbb{F}_{T}$ channel [58] and $0 l+$ monojet $+\mathscr{H}_{T}$ channel [59] by ATLAS.

Note that, we have used gluino masses around $1200 \mathrm{GeV}$ to estimate the relic density. However, the $t$ (and $u$ ) channel gluino exchange processes $\tilde{b}_{1} \tilde{b}_{1} \rightarrow b b$ contributes only about $10-25 \%$ to $\sigma_{\text {eff }}$ during DM freeze-out for our benchmark points. ${ }^{2}$ Even a large increment

\footnotetext{
${ }^{2}$ In $\tilde{b}_{1} \tilde{b}_{1}^{*} \rightarrow b \bar{b} s$ channel $g$ exchange contributes dominantly along with $t$ (and $u$ ) channel $\tilde{g}$ exchange
} 


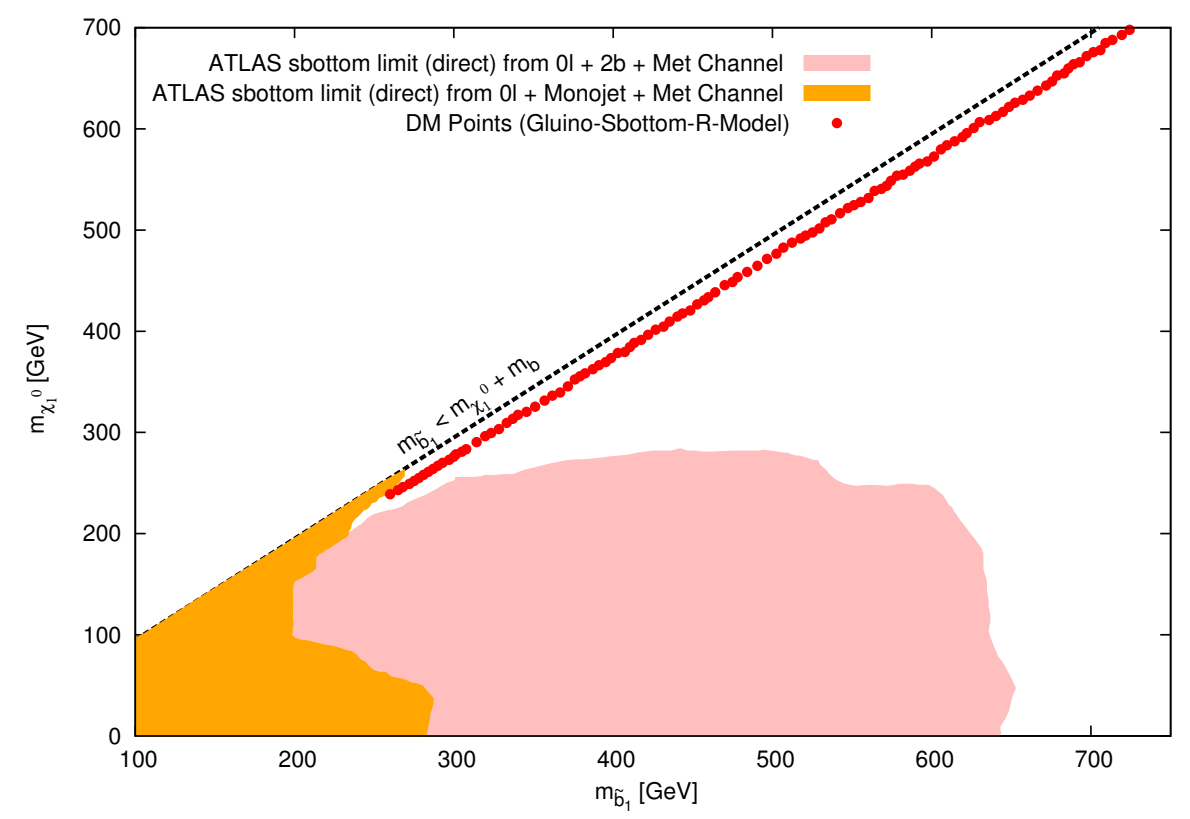

Figure 1: The red points give correct thermal relic density of $\widetilde{\chi}_{1}^{0}$ allowed by PLANCK [69] in Gluino-Sbottom-R-Model. Limits on $m_{\widetilde{b}_{1}}$ from direct $\widetilde{b}_{1}$ pair production from $0 l+$ monojet $+\mathscr{F}_{T}$ search [59] and $0 l+2 \mathrm{~b}+\mathbb{H}_{T}$ search [58] by ATLAS are presented by orange and pink shaded region respectively.

in $m_{\widetilde{g}}$, therefore, can be compensated by a corresponding reduction in the mass of the NLSP by a few $\mathrm{GeV}$, retaining the correct thermal relic abundance. We have checked this numerically using micrOMEGAs [80]. ${ }^{3}$

It should be further noted that we have assumed $|\mu|=1000 \mathrm{GeV}$. While reducing $|\mu|$ has no effect on the (2-body) decay of $\tilde{g}$; significant bino-higgsino mixing in $\widetilde{\chi}_{1}^{0}$ can be achieved. However, such mixing is quite constrained by the present data from LUX [81]. For a $300 \mathrm{GeV} \widetilde{\chi}_{1}^{0}$, we found it to be at most about $5-10 \%$. But the details of direct detection cross-section also depend on $\tan \beta$ and the heavy Higgs masses. Moreover, uncertainties from the estimation of the strange quark content of the nucleon affect the direct detection cross-section significantly; assuming the default values of strange quark content at low energies may turn out to be an oversimplification [80]. In the present context, its effect on the thermally averaged annihilation cross-section can again be compensated by changing the mass of the NLSP by a few $\mathrm{GeV}$, thus restoring the correct thermal relic abundance. It may thus be worthwhile to consider sbottom NLSP scenarios with low values of $|\mu|$ as well.

In a nutshell, we have considered various scenarios with a light $\tilde{b}_{1}$ NLSP (and also light $\tilde{t}_{1}$ in benchmarks BMP-B and BMP-C). In all these scenarios, the bino-like $\widetilde{\chi}_{1}^{0}$ provides a suitable Dark Matter candidate. While its dominantly bino-like nature assures that it

process. However, this channel contributes less than $1 \%$ to the thermally averaged annihilation cross-section.

${ }^{3}$ Note that, the mass difference between the LSP and the NLSP affects the contributions from the co-annihilating channels to $\sigma_{\text {eff }}$ exponentially. 


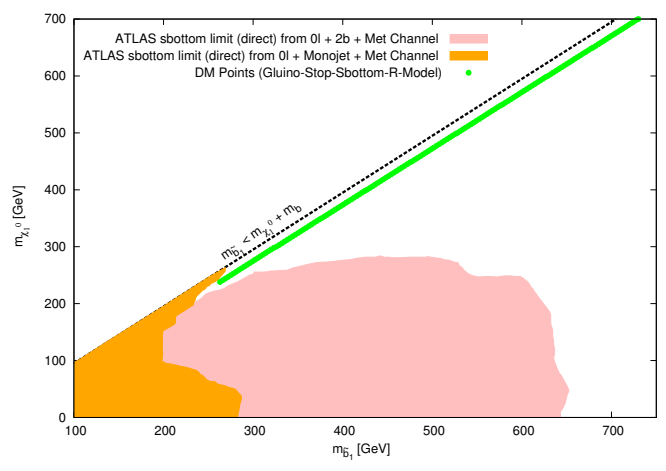

(a)

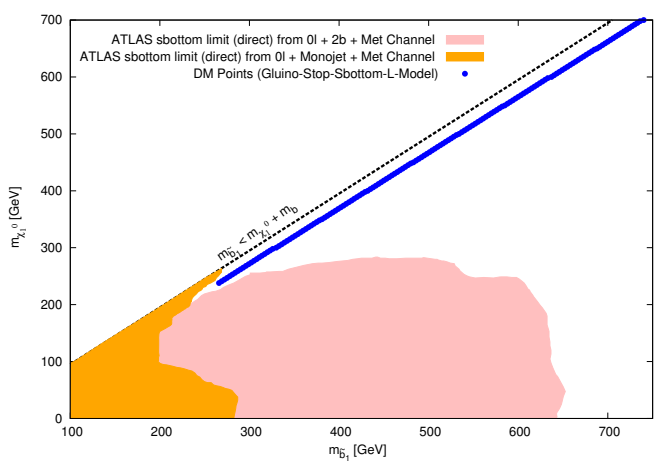

(b)

Figure 2: The green and blue points give correct thermal relic density of $\widetilde{\chi}_{1}^{0}$ allowed by PLANCK [69] in Gluino-Stop-Sbottom-R-Model (left panel) and Gluino-Stop-Sbottom-LModel (right panel) respectively (see text for details). Limits on $m_{\widetilde{b}_{1}}$ from direct $\widetilde{b}_{1}$ pair production from $0 l+$ monojet $+\not \mathbb{t}_{T}$ search [59] and $0 l+2 \mathrm{~b}+\mathbb{H}_{T}$ search [58] by ATLAS are presented by orange and pink shaded region respectively.

escapes the present direct searches, co-annihilation with $\tilde{b}_{1}$ plays a crucial role in achieving the right thermal relic density. In the next section, we will consider the lower bound on the mass of $\tilde{g}$ in these scenarios.

\section{Constraints on the $\widetilde{b}_{1}-\widetilde{g}$ sector in the $\widetilde{b}_{1}$ NLSP scenarios}

In this section we focus on $\widetilde{g}$ pair production in the sbottom NLSP scenarios described in the previous section and obtain the exclusion contours in the $m_{\widetilde{g}}-m_{\widetilde{b}_{1}}$ plane using the LHC data. For each point in the plot $m_{\widetilde{\chi}_{1}^{0}}$ is chosen in the narrow range consistent with the observed DM relic density. We also compute the limits on $m_{\widetilde{g}}$ for different models described in previous section and also for different BMPs introduced in table 1 and 2. Since there is no dedicated LHC search for this case our aim is to constrain the gluino mass utilizing the ATLAS model independent cross section bounds (see below) for final states similar to but not identical with the ones we are interested in. Our choice is guided by the fact that the final state coming from $\widetilde{g} \widetilde{g}$ pair production in all cases is expected to be enriched with $b$-jets and the generic signal will be jets $+\mathbb{F}_{T}$.

The ATLAS collaboration has updated their SUSY search results at $8 \mathrm{TeV}$ with $\mathcal{L} \sim$ $20 \mathrm{fb}^{-1}$ data in $n$-leptons $+m$-jets (with or without $b$ tagging) $+\mathbb{E}_{T}$ channel for different integral values of $n$ and $m$ and interpreted the results for various simplified models. Here we will concentrate mainly on the jets (at least $3 b$ jets $)+0-1 l(l=e, \mu)+\mathbb{E}_{T}[13]$ signal. The results were interpreted in a simplified model with light $\widetilde{g}$ and $\widetilde{b}_{1}$ for a fixed LSP mass: $m_{\widetilde{\chi}_{1}^{0}}=60 \mathrm{GeV}$. We have obtained new constraints for $\Delta m_{\widetilde{b}_{1}} \approx 30 \mathrm{GeV}$, which the hallmark of the $\widetilde{b}_{1}-\widetilde{\chi}_{1}^{0}$ coannihilation scenario. We have also considered the channel jets (no $b$ tagging $)+0 l(l=e, \mu)+\mathscr{f}_{T}[12]$. Although this analysis leads to weaker $m_{\widetilde{g}}$ limits 
in most cases of our interest, it yields the most stringent bounds for significantly smaller values of $\Delta m_{\widetilde{b}_{1}}$. Such choices, though disfavoured in the $\widetilde{b}_{1}-\widetilde{\chi}_{1}^{0}$ co- annihilation scenarios, can not be absolutely ruled out in view of the fact that DM production may be due to other mechanisms.

Next we will briefly review the above analyses by ATLAS. SUSY searches in the inclusive jets $+0 l+\mathbb{f}_{T}$ channel for $\mathcal{L}=20.3 \mathrm{fb}^{-1}$ at $8 \mathrm{TeV}$ have been presented in ref. [12]. Five inclusive analyses channels, labelled as A to E depending on jet multiplicities (from two to six respectively), are introduced. The relevant cuts have been described in table 1 of ref. [12]. Each of these channels are further classified as 'Tight','Medium' and 'Loose' based on the final cuts on the observables $\frac{\mathbb{F}_{T}}{m_{\mathrm{eff}}}$ and $m_{\mathrm{eff}}$ (incl.). ${ }^{4}$ Non-observation of any significant excess in each of these signal region leads to an upper limit on the number of events $\left(N_{B S M}\right)$ from any Beyond Standard Model (BSM) scenario. The observed upper limits on $N_{B S M}$ at $95 \%$ Confidence Level (CL) for signal regions SRA-Light, SRA-Medium, SRB-Medium, SRB-Tight, SRC-Medium, SRC-Tight, SRD, SRC-Loose, SRE-Tight, SREMedium, SRE-Loose are given by 1341, 51.3, 14.9, 6.7, 81.2, 2.4, 15.5, 92.4, 28.6, 8.3 respectively [12]. We use these (model independent) numbers to obtain new limits on $m_{\widetilde{g}}$.

ATLAS collaboration has also reported the search for strong sparticles in the multi$b$-jets final states with $\mathcal{L}=20.1 \mathrm{fb}^{-1}$ at $8 \mathrm{TeV}$ in [13]. Selection criteria for the signal regions are listed in table 1 and 2 of ref. [13]. In this analysis both $0 l$ (two signal regions) and at least one lepton (one signal region) channel are introduced. Signal regions are characterized by large $\mathbb{H}_{T}$ and at least four $(S R-0 l-4 j)$, six $(S R-1 l-6 j)$ or seven $(S R-0 l-7 j)$ jets which includes at least three $b$-tagged jets. Finally, these are further classified as $A / B / C$ depending on $\mathbb{E}_{T}$ and $m_{\text {eff }}$. The absence of any excess led to an upper limits on the number of signal events in each of these regions from ATLAS. In particular, for signal regions SR-0l-4j-A, SR-0l-4j-B, SR-0l-4j-C, SR-0l-7j-A, SR-0l-7j-B, SR-0l-7j-C, SR-11-6j-A, SR-11-6j-B, SR-11-6j-C these upper limits, at $95 \%$ CL, are given by 4.6, 6.7, $4.8,15.3,6.1,3.9,6.6,3.0,3.0$ respectively.

We adopt different selection criteria for various signal regions discussed above. For electron, muon and jet identification, reconstruction, isolation etc, we use the ATLAS prescription described in the respective analyses $[12,13] .{ }^{5}$ The $P_{T}$ dependent b-tagging efficiencies provided by ATLAS collaboration in ref. [82] have been used in our code. For validation, we reproduced the number of events in each of these signal regions, as obtained by ATLAS, for some benchmark points in refs. [12-15].

We then use PYTHIA (v6.428) [83] to generate the signal events in different channels from gluino pair production for different scenarios. The NLO cross-section for the $\widetilde{g} \widetilde{g}$ pair production is computed with PROSPINO 2.1 [84] using CTEQ6.6M PDF [85]. Finally we derive the new limits on gluino mass by comparing the computed number of events with the

\footnotetext{
${ }^{4} m_{\text {eff }}$ is defined as the scalar sum of the transverse momenta of the leading $\mathrm{N}$ jets which defines the signal region and $\mathbb{E}_{T} . m_{\text {eff }}$ (incl.) is defined as the scalar sum of the transverse momenta of the jets having $P_{T}$ greater than $40 \mathrm{GeV}$ and $\mathbb{F}_{T}$.

${ }^{5}$ Apart from the two analyses reported here we have also implemented the constraints from the hard single lepton channel $-1 l+$ jets $+\mathbb{E}_{T}$ channel [14] and the $2 l / 3 l+0-3$ bjets $+\mathbb{E}_{T}$ channel [15] in our code, but in most of the cases these channels only impose weaker constraints.
} 


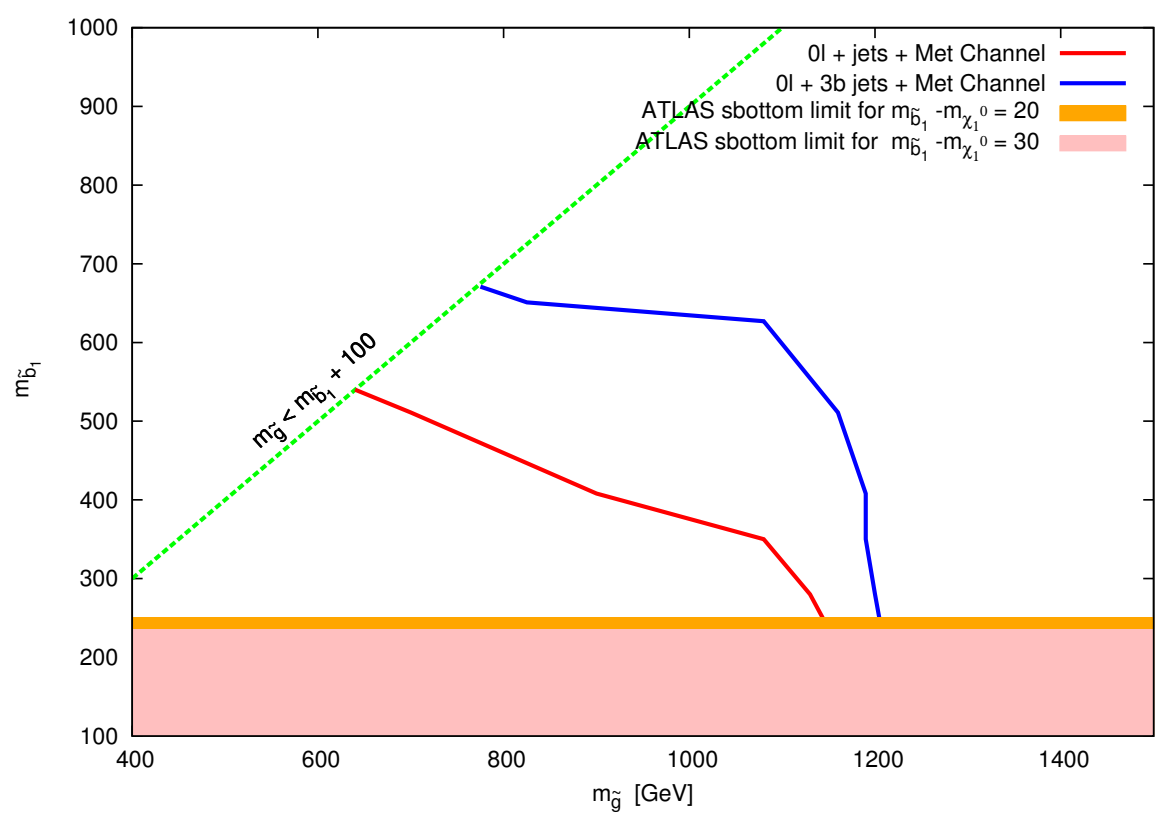

Figure 3: Limits on $m_{\widetilde{g}}$ in Gluino-Sbottom-R-Model from $\widetilde{g} \widetilde{g}$ pair production in $m_{\widetilde{g}}-m_{\widetilde{b}_{1}}$ plane from $0 l+j e t s+\mathbb{E}_{T}[12]$ and $0 l+j e t s(3 b)+\mathbb{F}_{T}$ channel [13] at $95 \% C L$ with $8 \mathrm{TeV} 20$ $\mathrm{fb}^{-1}$ ATLAS data. The shaded region are excluded from direct $\widetilde{b}_{1}$ pair production $[58,59]$ (see figure 1)

corresponding upper limits on $N_{B S M}$ in the relevant signal regions. The exclusion regions for Gluino-Sbottom-R-Model, thus obtained, are presented in figure 3.

In figure 3 each point gives correct DM relic density via sbottom coannihilation i.e., mass difference between $\widetilde{b}_{1}$ and $\widetilde{\chi}_{1}^{0}$ is in the range $25-35 \mathrm{GeV}$. We only consider the parameter space $m_{\widetilde{g}}>m_{\widetilde{b}_{1}}+100 \mathrm{GeV}$. If $m_{\widetilde{g}}$ is smaller then, as discussed in section 2 , the gluino contributes to DM relic production significantly leading to under abundant relic density. The shaded region in figure 3 is excluded by direct search for sbottom pairs for low $\Delta m_{\widetilde{b}_{1}}$.

The most stringent bound on $m_{\tilde{g}}$ comes from $0 l+j e t s(3 b)+\mathbb{F}_{T}$ channel [13] — for $m_{\widetilde{b}_{1}}$ around $500 \mathrm{GeV}$, gluino mass below $1.1-1.2 \mathrm{TeV}$ is excluded. The red and blue lines in figure 3 represents the exclusion contour from $0 l+j e t s+\mathbb{F}_{T}[12]$ and $0 l+j e t s(3 b)+\mathbb{H}_{T}$ channel [13]. Due to relatively soft b-jets from $\widetilde{b}_{1}$ decays, the signal in the sbottom NLSP scenario is sensitive to signal regions with low jet multiplicity. The two most effective signal regions are SRB-Medium [12] and SR-0l-4j-A [13] which require 3jets and 4jets respectively. In the Gluino-Stop-Sbottom-R-Model depending upon stop decay modes we consider three possibilities (see and table 1 and table 2). If the decay $\widetilde{t}_{1} \rightarrow c \widetilde{\chi}_{1}^{0}$ is the most dominant mode (see BMP-B1), then the gluino mass limits is expected to be rather weak. For our computations in this case we assume the mass relations $m_{\widetilde{b}_{1}}=m_{\widetilde{\chi}_{1}^{0}}+30 \mathrm{GeV}$ and $m_{\widetilde{t}_{1}}=m_{\widetilde{\chi}_{1}^{0}}+40 \mathrm{GeV}$. As already noted the relatively light $\widetilde{t}_{1}$ also contributes to DM production. We present the results in figure 4 for this model. Gluino limits in this model 


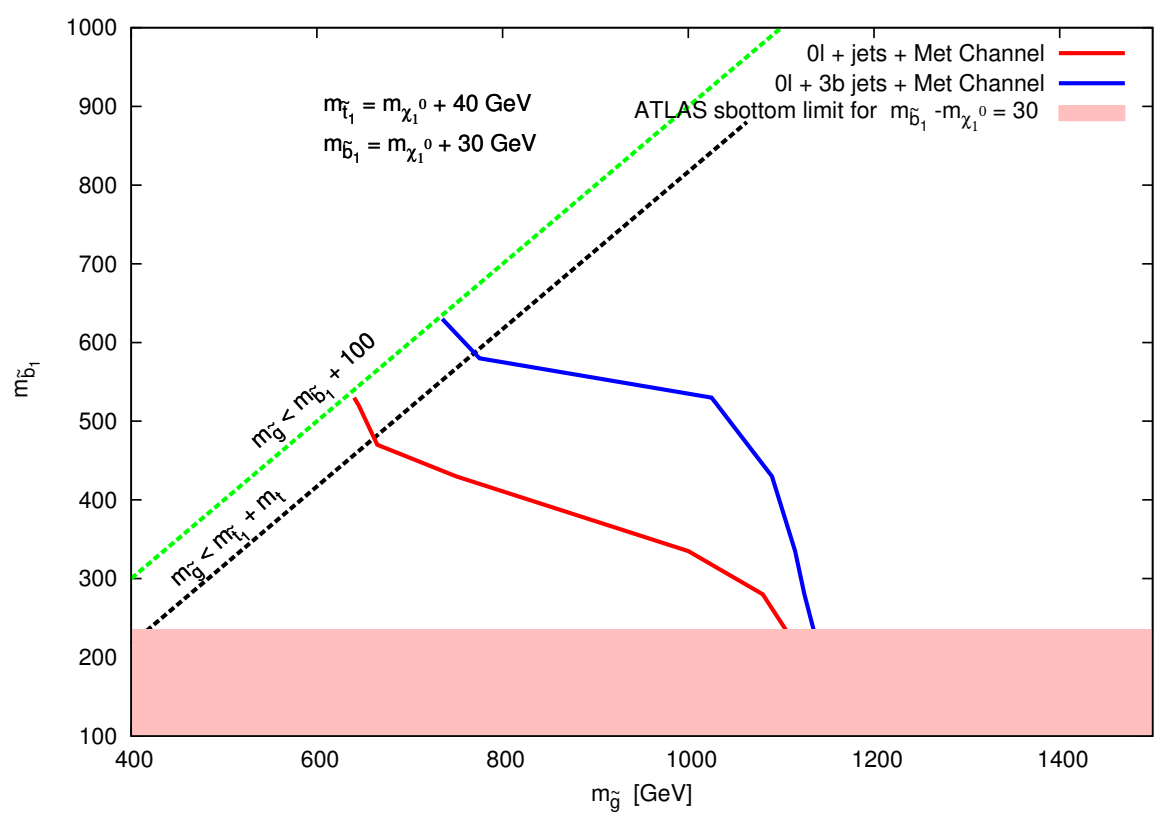

Figure 4: Limits on $m_{\widetilde{g}}$ in Gluino-Stop-Sbottom-R-Model from $\widetilde{g} \widetilde{g}$ pair production in $m_{\widetilde{g}}-m_{\widetilde{b}_{1}}$ plane from $0 l+j e t s+\mathbb{H}_{T}[12]$ and $0 l+j e t s(3 b)+\mathbb{H}_{T}$ channel [13] at $95 \%$ $C L$ with $8 \mathrm{TeV} 20 \mathrm{fb}^{-1}$ ATLAS data. Here $B R\left(\widetilde{b}_{1} \rightarrow b \widetilde{\chi}_{1}^{0}\right)=100 \%$ and $\widetilde{t}_{1} \rightarrow c \widetilde{\chi}_{1}^{0}$ dominates (for illustration see BMP-B1). The shaded region are excluded from direct $\widetilde{b}_{1}$ pair production $[58,59]$ (see figure 1)

are weaker than in figure 3 roughly by $100 \mathrm{GeV}$ in both channels. This is due to the fact that the gluino decays into $\widetilde{t}_{1} t$ with almost $50 \%$ BRs and effectively reduce the $0 l$ or bjet signal events. It may be noted that (see figure 4 ) when $\widetilde{g} \rightarrow \widetilde{t}_{1} t$ is kinematically disfavoured, then the limits tend to increase to some extent.

In the other scenarios where $\widetilde{t}_{1} \rightarrow t \widetilde{\chi}_{1}^{0}$ or $\widetilde{t}_{1} \rightarrow W \widetilde{b}_{1}$ dominates, there are extra sources of jets and tagged bjets. As a result the limits on $m_{\widetilde{g}}$ are pushed up. As the exclusion contour or mass limits lie between the results obtained in figure 3 and figure 4 , we have not presented any detailed figure for such scenarios. Instead we present the limits on $m_{\widetilde{g}}$ for the benchamark points (see table 3). As expected BMP-A gives the strongest limit $m_{\widetilde{g}} \gtrsim 1.2 \mathrm{TeV}$ and for BMP-B1 it reduced to $1.1 \mathrm{TeV}$. We note in passing that for BMPB2, BMP-B3 and BMP-C the signal contain multiple $W$ s which reduces the limits from the $0 l+j e t s+\mathbb{F}_{T}$ channel [12]. In such cases, however, the hard single lepton channel $\left(1 l+j e t s+\mathbb{F}_{T}\right)[14]$ puts more stringent bounds $\left(m_{\widetilde{g}}>1025-1050 \mathrm{GeV}\right)$.

If $\Delta m_{\widetilde{b}_{1}}$ happens to be much smaller than the values allowed by the DM relic density constraint, then $0 l+j e t s+\mathbb{H}_{T}$ channel yields a stronger lower bound on $m_{\widetilde{g}}$. For example, if $\Delta m_{\widetilde{b}_{1}}=10 \mathrm{GeV}$, a choice which cannot be strictly ruled out if the possibility of nonsupersymmetric DM is taken into account, the bound is about $1 \mathrm{TeV}$ in this channel. The corresponding limit from the $0 l+j e t s(3 b)+\not \mathbb{F}_{T}$ data is much weaker $(775 \mathrm{GeV})$. 


\begin{tabular}{|c|c|c|}
\hline \multirow{2}{*}{ Points } & \multicolumn{2}{|c|}{ Limit on $m_{\tilde{g}}(\mathrm{GeV})$} \\
\cline { 2 - 3 } & $0 l+$ jets $+\mathscr{F}_{T}[12]$ & $0 l+$ jets $(3 b)+\not_{T}[13]$ \\
\hline BMP-A & 1070 & 1195 \\
\hline BMP-B1 & 1000 & 1115 \\
\hline BMP-B2 & 845 & 1165 \\
\hline BMP-B3 & 850 & 1135 \\
\hline BMP-C & 850 & 1150 \\
\hline
\end{tabular}

Table 3: Limits on $m_{\widetilde{g}}$ using the ATLAS $0 l+j e t s+\mathbb{E}_{T}$ data $[12], 0 l+j e t s(3 b)+\mathbb{F}_{T}$ data [13] for the benchmarks.

In the $\widetilde{t}_{1}$ NLSP scenarios with correct DM relic density, the decays $\widetilde{b}_{1} W$ and $t \widetilde{\chi}_{1}^{0}$ are not allowed. However there are sufficiently large parameter space in the $\widetilde{b}_{1}$ NLSP scenario where these decays are allowed. These decays may provide novel signatures of this scenario during the LHC phase 2 experiments.

\section{Conclusion}

In view of the current interest in SUSY with light third generation squarks, we have studied the co-annihilating sbottom NLSP scenario and have delineated the parameter space consistent with the DM relic density constraint (see figure 1) when the NLSP is of $\mathrm{R}$-type with both of the stop mass eigenstates being rather heavy (the Gluino-Sbottom- $\mathrm{R}$ model). It easier to fit the observed Higgs mass in this case. It should also be stressed that in this scenario the co-annihilation cross section is relatively large since strong interactions partially contribute to it. As a result somewhat large $(25-35 \mathrm{GeV}) \Delta m_{\widetilde{b}_{1}}$ is consistent with the PLANCK data ( see eq. 2.1). This enhances the observability of the LHC signatures.

However, there are other options with an additional light $\widetilde{t}_{1}$ like the Gluino-SbottomStop-R model and the Gluino-Stop-Sbottom-L-Model (section 2). The allowed range of $\Delta m_{\widetilde{b}_{1}}$ is very similar to the one in the previous model (see figure 2). Some of the models predict spectacular LHC signatures with multiple tagged b-jets, $W^{\prime}$ s and reconstructable $t^{\prime} s$ over a large parameter space which should be searched for during phase 2 (section 2 ). These signals are not viable in the $\widetilde{t}_{1}$-NLSP scenario. The fraction of events with reconstructed $W$, top etc. can reveal the underlying scenarios including some hints on the mixing angles in the stop and sbottom sectors.

We note in passing that the $(g-2)_{\mu}$ anomaly $[86,87]$ can be easily resolved within the framework of the $\widetilde{b}_{1}$ NLSP scenario with relatively heavy electroweak sparticles. For example, if the sleptons are heavy, $m_{\widetilde{\chi}_{1}^{0}} \gtrsim 100 \mathrm{GeV}$, the constraint on the lighter chargino $\left(\widetilde{\chi}_{1}^{ \pm}\right)$mass from LHC is very weak and a large parameter space is consistent with the $(g-2)_{\mu}$ constraint. For lighter LSP there are stronger LHC constraints on the chargino mass; yet a reasonable parameter space compatible with the $(g-2)_{\mu}$ constraint is allowed (for a recent discussion see figure 8 and section 3.4 of ref. [73]). In the Gluino-Sbotom-R scenario the allowed $\widetilde{b}_{1}$-NLSP masses are bounded from below by the LHC lower limit $(260 \mathrm{GeV})$ from 
direct searches of coannihilating sbottom squarks. On the other hand $m_{\widetilde{b}_{1}}$ is bounded from above by the maximum chargino mass determined by the $(g-2)_{\mu}$ constraint, the LSP mass and the LHC searches for the electroweak sparticles. The same conclusion holds for Gluino-Sbottom-Stop-R model with $m_{\widetilde{b}_{1}}$ and $m_{\widetilde{t}_{1}}$ close together. In the variants of the $\widetilde{b}_{1}$ NLSP scenario with a heavier $\widetilde{t}_{1}$ the mode $\widetilde{t}_{1} \rightarrow b \widetilde{\chi}_{1}^{ \pm}$may open up.

There is no dedicated search for the $\widetilde{b}_{1}$ NLSP production from gluino decays as yet. We obtain new constraints on the $\widetilde{g}-\widetilde{b}_{1}$ sector in all scenarios using the existing LHC searches involving somewhat similar final states. We find that for $m_{s b o t}$ around $500 \mathrm{GeV}$ the limits on $m_{\widetilde{g}}$ in the $0 l+j e t s(3 b)+\mathbb{F}_{T}[13]$ channel vary in the range $1.1-1.2 \mathrm{TeV}$ in different scenarios (see figure 3, 4 and table 3). For higher $m_{\text {sbot }}$ the limit is degraded rapidly. On the other hand, for small $\Delta m_{\widetilde{b}_{1}}$ and $m_{\widetilde{g}} \approx 1 \mathrm{TeV}$, much stronger limits on the $\widetilde{b}_{1}$ mass is obtained via $\widetilde{g} \widetilde{g}$ pair production compared to that obtained from direct $\widetilde{b}_{1}$ searches. We find that the $0 l+j e t s+\mathscr{E}_{T}$ data generally yield somewhat weaker bounds. However, if the relic density constraint is relaxed and $\Delta m_{\widetilde{b}_{1}}$ is allowed to be smaller $(\approx 10 \mathrm{GeV})$ the strongest limit $(\approx 1 \mathrm{TeV})$ is obtained from the $0 l+j e t s+\mathbb{F}_{T}$ data. On the whole the gluino mass limit in the light sbottom scenario is about $1 \mathrm{TeV}$ irrespective of $\Delta m_{\widetilde{b}_{1}}$.

\section{Acknowledgments}

The work of A. Choudhury, A. Chatterjee and BM was partially supported by funding available from the Department of Atomic Energy, Government of India, for the Regional Centre for Accelerator-based Particle Physics (RECAPP), Harish-Chandra Research Institute. AD acknowledges the award of a Senior Scientist position by the Indian National Science Academy. AD also thanks RECAPP, HRI for the hospitality during the initial phase of the project.

Open Access. This article is distributed under the terms of the Creative Commons Attribution License (CC-BY 4.0), which permits any use, distribution and reproduction in any medium, provided the original author(s) and source are credited.

\section{References}

[1] H.P. Nilles, Supersymmetry, Supergravity and Particle Physics, Phys. Rept. 110 (1984) 1 [INSPIRE].

[2] H.E. Haber and G.L. Kane, The Search for Supersymmetry: Probing Physics Beyond the Standard Model, Phys. Rept. 117 (1985) 75 [InSPIRE].

[3] S.P. Martin, A Supersymmetry primer, Adv. Ser. Direct. High Energy Phys. 21 (2010) 1 [hep-ph/9709356] [INSPIRE].

[4] J. Wess and J. Bagger, Supersymmetry and Supergravity, 2nd ed., Princeton University Press, Princeton U.S.A. (1991).

[5] M. Drees, P. Roy and R. M. Godbole, Theory and Phenomenology of Sparticles, World Scientific, Singapore (2005). 
[6] G. Jungman, M. Kamionkowski and K. Griest, Supersymmetric dark matter, Phys. Rept. 267 (1996) 195 [hep-ph/9506380] [INSPIRE].

[7] W.L. Freedman and M.S. Turner, Measuring and understanding the universe, Rev. Mod. Phys. 75 (2003) 1433 [astro-ph/0308418] [inSPIRE].

[8] L. Roszkowski, Particle dark matter: A Theorist's perspective, Pramana 62 (2004) 389 [hep-ph/0404052] [INSPIRE].

[9] G. Bertone, D. Hooper and J. Silk, Particle dark matter: Evidence, candidates and constraints, Phys. Rept. 405 (2005) 279 [hep-ph/0404175] [INSPIRE].

[10] H. Baer and X. Tata, Dark matter and the LHC, arXiv:0805.1905 [INSPIRE].

[11] A. Datta, B. Mukhopadhyaya and A. Raychaudhuri (eds.), Physics at the Large Hadron Collider, INSA, Springer Germany (2009).

[12] ATLAS collaboration, Search for squarks and gluinos with the ATLAS detector in final states with jets and missing transverse momentum and $20.3 \mathrm{fb}^{-1}$ of $\sqrt{s}=8$ TeV proton-proton collision data, ATLAS-CONF-2013-047 (2013).

[13] ATLAS collaboration, Search for strong production of supersymmetric particles in final states with missing transverse momentum and at least three b-jets using $20.1 \mathrm{fb}^{-1}$ of $\mathrm{pp}$ collisions at $\sqrt{s}=8 \mathrm{TeV}$ with the ATLAS Detector., ATLAS-CONF-2013-061 (2013).

[14] ATLAS collaboration, Search for squarks and gluinos in events with isolated leptons, jets and missing transverse momentum at $\sqrt{s}=8 \mathrm{TeV}$ with the ATLAS detector,

ATLAS-CONF-2013-062 (2013).

[15] ATLAS collaboration, Search for supersymmetry at $\sqrt{s}=8$ TeV in final states with jets and two same-sign leptons or three leptons with the ATLAS detector, JHEP 06 (2014) 035 [arXiv: 1404.2500] [INSPIRE].

[16] CMS collaboration, Search for top-squark pair production in the single-lepton final state in pp collisions at $\sqrt{s}=8 \mathrm{TeV}$, Eur. Phys. J. C 73 (2013) 2677 [arXiv:1308.1586] [inSPIRE].

[17] CMS collaboration, Search for supersymmetry in pp collisions at $\sqrt{s}=8 \mathrm{TeV}$ in events with a single lepton, large jet multiplicity and multiple b jets, Phys. Lett. B 733 (2014) 328 [arXiv: 1311.4937] [INSPIRE].

[18] CMS collaboration, Search for new physics in events with same-sign dileptons and jets in pp collisions at $\sqrt{s}=8 \mathrm{TeV}$, JHEP 01 (2014) 163 [arXiv:1311.6736] [INSPIRE].

[19] CMS collaboration, Search for new physics in the multijet and missing transverse momentum final state in proton-proton collisions at $\sqrt{s}=8 \mathrm{TeV}$, JHEP 06 (2014) 055 [arXiv: 1402.4770] [INSPIRE].

[20] T.J. LeCompte and S.P. Martin, Compressed supersymmetry after 1/fb at the Large Hadron Collider, Phys. Rev. D 85 (2012) 035023 [arXiv:1111.6897] [InSPIRE].

[21] H.K. Dreiner, M. Krämer and J. Tattersall, How low can SUSY go? Matching, monojets and compressed spectra, Europhys. Lett. 99 (2012) 61001 [arXiv:1207.1613] [INSPIRE].

[22] B. Bhattacherjee and K. Ghosh, Degenerate SUSY search at the $8 \mathrm{TeV} L H C$, arXiv: 1207.6289 [INSPIRE].

[23] B. Bhattacherjee, A. Choudhury, K. Ghosh and S. Poddar, Compressed supersymmetry at 14 TeV LHC, Phys. Rev. D 89 (2014) 037702 [arXiv:1308.1526] [InSPIRE]. 
[24] T. Cohen, T. Golling, M. Hance, A. Henrichs, K. Howe et al., SUSY Simplified Models at 14, 33 and 100 TeV Proton Colliders, JHEP 04 (2014) 117 [arXiv:1311.6480] [InSPIRE].

[25] S. Mukhopadhyay, M.M. Nojiri and T.T. Yanagida, Compressed SUSY search at the $13 \mathrm{TeV}$ LHC using kinematic correlations and structure of ISR jets, JHEP 1410 (2014) 12 [arXiv: 1403.6028] [INSPIRE].

[26] B. Fuks, P. Richardson and A. Wilcock, Pinning down compressed supersymmetric scenarios with monotop probes, arXiv:1408.3634 [INSPIRE].

[27] C. Brust, A. Katz, S. Lawrence and R. Sundrum, SUSY, the Third Generation and the LHC, JHEP 03 (2012) 103 [arXiv:1110.6670] [INSPIRE].

[28] N. Desai and B. Mukhopadhyaya, Constraints on supersymmetry with light third family from LHC data, JHEP 05 (2012) 057 [arXiv:1111.2830] [INSPIRE].

[29] Z. Han, A. Katz, D. Krohn and M. Reece, (Light) Stop Signs, JHEP 08 (2012) 083 [arXiv: 1205.5808] [INSPIRE].

[30] X.-J. Bi, Q.-S. Yan and P.-F. Yin, Light stop/sbottom pair production searches in the NMSSM, Phys. Rev. D 87 (2013) 035007 [arXiv: 1209. 2703] [INSPIRE].

[31] B. Bhattacherjee, S.K. Mandal and M. Nojiri, Top Polarization and Stop Mixing from Boosted Jet Substructure, JHEP 03 (2013) 105 [arXiv: 1211.7261] [INSPIRE].

[32] M. Carena, S. Gori, N.R. Shah, C.E.M. Wagner and L.-T. Wang, Light Stops, Light Staus and the $125 \mathrm{GeV}$ Higgs, JHEP 08 (2013) 087 [arXiv: 1303.4414] [INSPIRE].

[33] P. Agrawal and C. Frugiuele, Mixing stops at the LHC, JHEP 01 (2014) 115 [arXiv: 1304.3068] [INSPIRE].

[34] Y. Bai, H.-C. Cheng, J. Gallicchio and J. Gu, A Toolkit of the Stop Search via the Chargino Decay, JHEP 08 (2013) 085 [arXiv: 1304.3148] [INSPIRE].

[35] C. Han, K.-i. Hikasa, L. Wu, J.M. Yang and Y. Zhang, Current experimental bounds on stop mass in natural SUSY, JHEP 10 (2013) 216 [arXiv:1308.5307] [INSPIRE].

[36] D. Goncalves, D. Lopez-Val, K. Mawatari and T. Plehn, Automated third generation squark production to next-to-leading order, Phys. Rev. D 90 (2014) 075007 [arXiv:1407.4302] [INSPIRE].

[37] M.A. Ajaib, T. Li and Q. Shafi, Stop-Neutralino Coannihilation in the Light of LHC, Phys. Rev. D 85 (2012) 055021 [arXiv:1111.4467] [InSPIRE].

[38] A. Choudhury and A. Datta, New limits on top squark NLSP from LHC $4.7 \mathrm{fb}^{-1}$ data, Mod. Phys. Lett. A 27 (2012) 1250188 [arXiv:1207.1846] [INSPIRE].

[39] K. Ghosh et al., Top jets as a probe of degenerate stop-NLSP LSP scenario in the framework of CMSSM, Phys. Rev. Lett. 110 (2013) 141801 [arXiv:1207.2429] [INSPIRE].

[40] Z.-H. Yu, X.-J. Bi, Q.-S. Yan and P.-F. Yin, Detecting light stop pairs in coannihilation scenarios at the LHC, Phys. Rev. D 87 (2013) 055007 [arXiv:1211.2997] [INSPIRE].

[41] K. Krizka, A. Kumar and D.E. Morrissey, Very Light Scalar Top Quarks at the LHC, Phys. Rev. D 87 (2013) 095016 [arXiv: 1212.4856] [INSPIRE].

[42] G. Bélanger, D. Ghosh, R. Godbole, M. Guchait and D. Sengupta, Probing the flavor violating scalar top quark signal at the LHC, Phys. Rev. D 89 (2014) 015003 [arXiv: 1308.6484] [INSPIRE]. 
[43] A. Belyaev, T. Lastovicka, A. Nomerotski and G. Lastovicka-Medin, Discovering Bottom Squark Co-annihilation at ILC, Phys. Rev. D 81 (2010) 035011 [arXiv:0912.2411] [INSPIRE].

[44] M. Adeel Ajaib, T. Li and Q. Shafi, Searching for NLSP Sbottom at the LHC, Phys. Lett. B 701 (2011) 255 [arXiv:1104.0251] [INSPIRE].

[45] I. Gogoladze, S. Raza and Q. Shafi, Neutralino-Sbottom Coannihilation in SU(5), JHEP 03 (2012) 054 [arXiv:1111.6299] [INSPIRE].

[46] E. Alvarez and Y. Bai, Reach the Bottom Line of the Sbottom Search, JHEP 08 (2012) 003 [arXiv: 1204.5182] [INSPIRE].

[47] M. Badziak, Yukawa unification in SUSY SO(10) in light of the LHC Higgs data, Mod. Phys. Lett. A 27 (2012) 1230020 [arXiv:1205.6232] [INSPIRE].

[48] J. Alwall, J.L. Feng, J. Kumar and S. Su, B's with Direct Decays: Tevatron and LHC Discovery Prospects in the $b \bar{b}+$ MET Channel, Phys. Rev. D 84 (2011) 074010 [arXiv:1107.2919] [INSPIRE].

[49] H.M. Lee, V. Sanz and M. Trott, Hitting sbottom in natural SUSY, JHEP 05 (2012) 139 [arXiv:1204.0802] [INSPIRE].

[50] D. Ghosh and D. Sengupta, Searching the sbottom in the four lepton channel at the LHC, Eur. Phys. J. C 73 (2013) 2342 [arXiv:1209.4310] [inSPIRE].

[51] A. Chakraborty, D.K. Ghosh, D. Ghosh and D. Sengupta, Stop and sbottom search using dileptonic $M_{T 2}$ variable and boosted top technique at the LHC, JHEP 10 (2013) 122 [arXiv:1303.5776] [INSPIRE].

[52] J.S. Hagelin, S. Kelley and T. Tanaka, Supersymmetric flavor changing neutral currents: Exact amplitudes and phenomenological analysis, Nucl. Phys. B 415 (1994) 293 [INSPIRE].

[53] T. Ibrahim and P. Nath, The Neutron and the lepton EDMs in MSSM, large CP-violating phases and the cancellation mechanism, Phys. Rev. D 58 (1998) 111301 [Erratum ibid. D 60 (1999) 099902] [hep-ph/9807501] [INSPIRE].

[54] M. Pospelov and A. Ritz, Electric dipole moments as probes of new physics, Annals Phys. 318 (2005) 119 [hep-ph/0504231] [INSPIRE].

[55] MSSM Working Group collaboration, A. Djouadi et al., The Minimal supersymmetric standard model: Group summary report, hep-ph/9901246 [INSPIRE].

[56] ATLAS collaboration, Search for direct pair production of the top squark in all-hadronic final states in proton-proton collisions at $\sqrt{s}=8 \mathrm{TeV}$ with the ATLAS detector, JHEP 09 (2014) 015 [arXiv: 1406.1122] [INSPIRE].

[57] ATLAS collaboration, Search for top squark pair production in final states with one isolated lepton, jets and missing transverse momentum in $\sqrt{s}=8 \mathrm{TeV}$ pp collisions with the ATLAS detector, JHEP 11 (2014) 118 [arXiv:1407.0583] [INSPIRE].

[58] ATLAS collaboration, Search for direct third-generation squark pair production in final states with missing transverse momentum and two b-jets in $\sqrt{s}=8 \mathrm{TeV}$ pp collisions with the ATLAS detector, JHEP 10 (2013) 189 [arXiv:1308.2631] [INSPIRE].

[59] ATLAS collaboration, Search for pair-produced third-generation squarks decaying via charm quarks or in compressed supersymmetric scenarios in pp collisions at $\sqrt{s}=8 \mathrm{TeV}$ with the ATLAS detector, Phys. Rev. D 90 (2014) 052008 [arXiv:1407.0608] [InSPIRE]. 
[60] J. Hisano, K. Kawagoe, R. Kitano and M.M. Nojiri, Scenery from the top: Study of the third generation squarks at CERN LHC, Phys. Rev. D 66 (2002) 115004 [hep-ph/0204078] [INSPIRE].

[61] J. Hisano, K. Kawagoe and M.M. Nojiri, A Detailed study of the gluino decay into the third generation squarks at the CERN LHC, Phys. Rev. D 68 (2003) 035007 [hep-ph/0304214] [INSPIRE].

[62] N. Desai and B. Mukhopadhyaya, Signals of supersymmetry with inaccessible first two families at the Large Hadron Collider, Phys. Rev. D 80 (2009) 055019 [arXiv:0901.4883] [INSPIRE].

[63] N. Bhattacharyya, A. Choudhury and A. Datta, SUSY signals with small and large trilinear couplings at the LHC $7 \mathrm{TeV}$ runs and neutralino dark matter, Phys. Rev. D 83 (2011) 115025 [arXiv: 1104.0333] [inSPIRE].

[64] P. Bandyopadhyay and B. Bhattacherjee, Boosted top quarks in supersymmetric cascade decays at the LHC, Phys. Rev. D 84 (2011) 035020 [arXiv: 1012.5289] [INSPIRE].

[65] N. Bhattacharyya, A. Choudhury and A. Datta, Low mass neutralino dark matter in mSUGRA and more general models in the light of LHC data, Phys. Rev. D 84 (2011) 095006 [arXiv: 1107.1997] [INSPIRE].

[66] A. Datta and S. Niyogi, Entangled System of Squarks from the Third Generation at the Large Hadron Collider, arXiv: 1111.0200 [INSPIRE].

[67] A. Choudhury and A. Datta, Many faces of low mass neutralino dark matter in the unconstrained MSSM, LHC data and new signals, JHEP 06 (2012) 006 [arXiv:1203.4106] [INSPIRE].

[68] S. Bhattacharya, S. Chakdar, K. Ghosh and S. Nandi, Non-universal SUGRA at LHC: Prospects and Discovery Potential, Phys. Rev. D 89 (2014) 015004 [arXiv:1309.0036] [INSPIRE].

[69] Planck collaboration, Planck 2013 results. XVI. Cosmological parameters, Astron. Astrophys. 571 (2014) A16 [arXiv:1303.5076] [INSPIRE].

[70] K. Griest and D. Seckel, Three exceptions in the calculation of relic abundances, Phys. Rev. D 43 (1991) 3191 [inSPIRE].

[71] J. Edsjo and P. Gondolo, Neutralino relic density including coannihilations, Phys. Rev. D 56 (1997) 1879 [hep-ph/9704361] [INSPIRE].

[72] A. Choudhury and A. Datta, Neutralino dark matter confronted by the LHC constraints on Electroweak SUSY signals, JHEP 09 (2013) 119 [arXiv:1305.0928] [INSPIRE].

[73] M. Chakraborti, U. Chattopadhyay, A. Choudhury, A. Datta and S. Poddar, The Electroweak Sector of the pMSSM in the Light of LHC - $8 \mathrm{TeV}$ and Other Data, JHEP 07 (2014) 019 [arXiv:1404.4841] [INSPIRE].

[74] A. Djouadi, J.-L. Kneur and G. Moultaka, SuSpect: A Fortran code for the supersymmetric and Higgs particle spectrum in the MSSM, Comput. Phys. Commun. 176 (2007) 426 [hep-ph/0211331] [INSPIRE].

[75] ATLAS collaboration, Observation of a new particle in the search for the Standard Model Higgs boson with the ATLAS detector at the LHC, Phys. Lett. B 716 (2012) 1 [arXiv: 1207.7214] [INSPIRE]. 
[76] CMS collaboration, Observation of a new boson at a mass of 125 GeV with the CMS experiment at the LHC, Phys. Lett. B 716 (2012) 30 [arXiv:1207.7235] [INSPIRE].

[77] ATLAS collaboration, Updated coupling measurements of the Higgs boson with the ATLAS detector using up to $25 \mathrm{fb}^{-1}$ of proton-proton collision data, ATLAS-CONF-2014-009 (2014).

[78] CMS collaboration, Precise determination of the mass of the Higgs boson and studies of the compatibility of its couplings with the standard model, CMS-PAS-HIG-14-009 (2014).

[79] A. Djouadi, M.M. Muhlleitner and M. Spira, Decays of supersymmetric particles: The Program SUSY-HIT (SUspect-SdecaY-HDECAY-InTerface), Acta Phys. Polon. B 38 (2007) 635 [hep-ph/0609292] [INSPIRE].

[80] G. Bélanger, F. Boudjema, A. Pukhov and A. Semenov, MicrOMEGAs 3 : A program for calculating dark matter observables, Comput. Phys. Commun. 185 (2014) 960 [arXiv: 1305.0237] [INSPIRE].

[81] LUX collaboration, D.S. Akerib et al., First results from the LUX dark matter experiment at the Sanford Underground Research Facility, Phys. Rev. Lett. 112 (2014) 091303 [arXiv: 1310.8214] [INSPIRE].

[82] ATLAS collaboration, Measurement of the b-tag Efficiency in a Sample of Jets Containing Muons with $5 \mathrm{fb}^{-1}$ of Data from the ATLAS Detector, ATLAS-CONF-2012-043 (2012).

[83] T. Sjöstrand, S. Mrenna and P.Z. Skands, PYTHIA 6.4 Physics and Manual, JHEP 05 (2006) 026 [hep-ph/0603175] [INSPIRE].

[84] W. Beenakker, R. Hopker, M. Spira and P.M. Zerwas, Squark and gluino production at hadron colliders, Nucl. Phys. B 492 (1997) 51 [hep-ph/9610490] [INSPIRE].

[85] P.M. Nadolsky et al., Implications of CTEQ global analysis for collider observables, Phys. Rev. D 78 (2008) 013004 [arXiv:0802.0007] [InSPIRE].

[86] Muon G-2 collaboration, G.W. Bennett et al., Final Report of the Muon E821 Anomalous Magnetic Moment Measurement at BNL, Phys. Rev. D 73 (2006) 072003 [hep-ex/0602035] [INSPIRE].

[87] B.L. Roberts, Status of the Fermilab Muon $(g-2)$ Experiment, Chin. Phys. C 34 (2010) 741 [arXiv: 1001.2898] [INSPIRE]. 